\title{
States and energies of the periodic Hubbard model at half filling
}

\author{
Gabriel Alvarez ${ }^{1}$, Francisco Guil \\ Departamento de Física Teórica, Facultad de Ciencias Fisicas, Universidad Complutense. \\ 28040 Madrid. Spain
}

Received 29 March 1996; accepted 13 May 1996

\begin{abstract}
The representation of the periodic Hubbard model in the Clifford algebra leads to explicit expressions for several families of non-trivial half-filled states in any number of dimensions. A generalization of these expressions explains the structure of the spectrum of the general Hubbard hamiltonian.
\end{abstract}

PACS: 71.10.Fd; 02.20.Qs

Keywords: Hubbard model; Representation theory

\section{Introduction}

The main goal of this paper is the systematic study of certain families of non-trivial half-filled states in the standard one-band periodic Hubbard model [1-3]. Apart from the one-dimensional case, solved by Lieb and Wu [4] within the framework of the Bethe ansatz and for which there is an extensive literature [1-3], few exact results on the spectrum of the Hubbard hamiltonian are known. Particularly noteworthy among these are the spin theorems of Lieb [6], the " $\eta$-pairing" states found by Yang [5] in bipartite $d$-dimensional lattices, and the special lattices studied by Mielke and Tasaki [7].

In a recent paper [8] we introduced a new representation of the Hubbard model in which half of the variables are eliminated, and the states become operators in a Clifford algebra. This representation, especially suited to describe complex many-particle states

\footnotetext{
${ }^{1}$ E-mail: alvarez@lisa.fis.ucm.es. 
in simple terms, immediately suggested several families of non-trivial eigenstates at half filling. In the present paper we show how these states can be systematically understood, derive explicit equations for both the eigenvalues and the eigenvectors, and show how more general states fit the same pattern. We stress that our results are independent of the dimension and of the bipartite character of the lattice, although in this case there are additional spectral symmetries as discussed by Yang and Zhang [9].

The layout of the paper is as follows. In Section 2, after recalling briefly the Clifford algebra representation of the Hubbard model, we study the connection between spin and invariant modules, and discuss spectral symmetries of the eigenvalues. Section 3 is devoted to periodic lattices. We switch to the momentum representation and obtain explicit expressions for the eigenvalues and eigenvectors quoted above. Finally, in Section 4 we study the structure of the space of states and show how the expressions we found in Section 3 are particular instances of more general equations that can be applied to understand the structure of the spectrum of the general Hubbard hamiltonian.

\section{The space of states as an exterior algebra}

\subsection{Realizations of the Hilbert space}

The Hilbert space of a system of interacting electrons moving on a graph $L$ with $\ell$ sites can be identified with the direct product of two copies of the exterior algebra $A(E)$ of the space of states $E$ of a single spinless particle moving on the same graph $L$. To realize this identification, we fix an orthonormal basis $\mathcal{B}=\left\{x_{1}, \ldots, x_{\ell}\right\}$ of $E$ such that a spin-up or spin-down electron (whose space of states is isomorphic to $E \oplus E$ ) localized at the site $i$ is described by $\left(x_{i}, 0\right)$ or $\left(0, x_{i}\right)$ respectively, and apply the fundamental property of the exterior algebra with respect to the direct sum of subspaces,

$$
\mathcal{H}=\Lambda(E \oplus E) \cong \Lambda(E) \otimes \Lambda(E)
$$

We will denote with the same symbol the vector $u \in E$ and the exterior-multiplication operator by $u$ in $\Lambda(E)$. The adjoint of $u$ with respect to the scalar product induced in $\Lambda(E)$ acts as an inner derivation and will be denoted by $\partial / \partial u$. In particular, the operators associated to the basis elements $x_{i} \in \mathcal{B}$ satisfy the canonical anticommutation relations

$$
\begin{aligned}
x_{i} x_{j}+x_{j} x_{i} & =0, \\
x_{i} \frac{\partial}{\partial x_{j}}+\frac{\partial}{\partial x_{j}} x_{i} & =\delta_{i j} .
\end{aligned}
$$

The kinetic energy of the Hubbard model is specified by a self-adjoint linear operator $t$ in $E$ whose matrix $t_{i j}$ in the basis $\mathcal{B}$ (the "hopping matrix") has vanishing diagonal elements. The definition 


$$
K=\sum_{i, j=1}^{\ell} t_{i j} x_{i} \frac{\partial}{\partial x_{j}}
$$

extends $t$ to the whole exterior algebra $\Lambda(E)$, and the kinetic-energy operator associated to $t$ is the representation $T$ of $t$ in $\Lambda(E) \otimes \Lambda(E)$, i.e. the operator $T(u \otimes v)=K u \otimes$ $v+u \otimes K v$. Similarly, the potential energy $W$ of the Hubbard model can be written in terms of the operators $N_{i}(u \otimes v)=n_{i} u \otimes v+u \otimes n_{i} v$, where $n_{i}=x_{i} \partial / \partial x_{i}$ represents the elementary matrix $E_{i i}$ in $\Lambda(E)$,

$$
W=\frac{U}{2} \sum_{i=1}^{\ell}\left(N_{i}-1\right)^{2}-\frac{\ell U}{4}
$$

Note that "pairs" ( $N_{i}=2$ or doubly occupied sites) and "holes" ( $N_{i}=0$ or empty sites) contribute the same amount to the potential energy of a configuration. The complete Hubbard hamiltonian is

$$
H=T+W
$$

In Ref. [8] we discussed at length an equivalent formulation that follows from the isomorphism $\mathcal{H} \cong C(\ell)=\Lambda(E) \otimes \Lambda(E)^{*}$ between the Hilbert space $\mathcal{H}$ and the complex Clifford algebra $C(\ell)$. There are two particularly useful realizations of this isomorphism. To obtain the first one, we pick a volume element $\Omega \in A^{\ell}(E)$ and identify $u \otimes v$ with the linear operator $u \Omega^{+} v$. The Hubbard hamiltonian becomes

$$
H(X)=[K, X]+U \Delta_{0}(X)-\frac{\ell U}{4} X,
$$

where

$$
\Delta_{0}(X)=\frac{1}{2} \sum_{i=1}^{\ell}\left[n_{i},\left[n_{i}, X\right]\right]
$$

is minus the Casimir operator of the Cartan subalgebra $g l_{0}$ of $g l(\ell)$ that acts on $E$ by diagonal transformations in the basis $\mathcal{B}$. In this realization the elementary block $\Lambda^{j}(E) \otimes A^{k}(E)^{*}$ of $C(\ell)$ with respect to the decomposition $A(E)=\bigoplus_{k=0}^{\ell} \Lambda^{k}(E)$ is precisely the spin module with $j$ spin-up electrons and $\ell-k$ spin-down electrons.

The second realization discussed in Ref. [8] follows from the isomorphism $\Lambda(E) \otimes$ $\Lambda(E)^{*} \cong \Lambda(E) \otimes \Lambda\left(E^{*}\right)$, which leads to the same action of the hamiltonian but to a different decomposition of $C(\ell)$ into $g l(\ell)$ modules,

$$
C(\ell) \cong \bigoplus_{A, B=0}^{\ell} \Gamma_{A B}
$$

where

$$
\Gamma_{A B}=\Lambda^{A}(E) \otimes \Lambda^{B}\left(E^{*}\right)=\mathbf{C}\left\{x^{\alpha} \frac{\partial^{\beta}}{\partial x^{\beta}},|\alpha|=A,|\beta|=B\right\} .
$$


Here $\alpha=\left(\alpha_{1}, \ldots, \alpha_{\ell}\right)$ and $\beta=\left(\beta_{1}, \ldots, \beta_{\ell}\right)\left(\alpha_{i}, \beta_{i} \in\{0,1\}\right)$ are multi-indices, and $x^{\alpha}=x_{1}^{\alpha_{1}} \ldots x_{\ell}^{\alpha_{\ell}}$ as usual, with a similar equation for $\partial^{\beta} / \partial x^{\beta}$. The states $x^{\alpha} \partial^{\beta} / \partial x^{\beta} \in$ $\Gamma_{A B}$ have $A(\alpha, \beta)$ pairs, $B(\alpha, \beta)$ holes, $A-B+\ell$ particles, and are represented by matrices in the $(B-A)$ th diagonal above (or below) the main diagonal in the block decomposition.

As a final remark on the general properties of the Hubbard model we mention that the action of the unitary group on $E$ preserves the structure of the Hubbard hamiltonian. We have seen that the potential energy is determined by the Casimir operator $-\Delta_{0}$ of the Cartan subalgebra $g l_{0}$, and that the kinetic energy is the representation of a hopping matrix $t$ that belongs to the image of the adjoint action of $g l_{0}$. Our remark is a direct consequence of the fact that any two Cartan subalgebras are conjugate (see Ref. [10]).

\subsection{Spin}

There is a close connection between the $g l(\ell)$ modules $\Gamma_{A B}$ and the spin algebra $g l(2) \cong \mathbf{C}\left\{N, S_{+}, S_{-}, S_{z}\right\}$ generated by $S_{+}: \Gamma_{A B} \rightarrow \Gamma_{A+1, B+1}$,

$$
S_{+}\left(x^{\alpha} \frac{\partial^{\beta}}{\partial x^{\beta}}\right)=(-1)^{\ell+1} x^{\alpha} n \frac{\partial^{\beta}}{\partial x^{\beta}},
$$

where

$$
n=\sum_{i=1}^{\ell} x_{i} \frac{\partial}{\partial x_{i}}
$$

represents the identity matrix in $A(E), S_{-}=S_{+}^{+}, S_{z}=\frac{1}{2}\left[S_{+}, S_{-}\right]$, and the number of particles operator

$$
N(X)=[n, X]+\ell X .
$$

Each $g l(\ell)$ module $\Gamma_{A B}$ decomposes into a direct sum of irreducible highest-weight modules with respect to the weight structure defined by the unitary diagonal matrices in the fixed basis $\mathcal{B}$. Furthermore, the operator $S_{+}$preserves this weight structure and maps irreducible modules to irreducible modules, because $n$ commutes with every element of $g l(\ell)$. For $A+B \geqslant \ell$ the kernel of $S_{+}$is an irreducible module, and the isomorphism $\Gamma_{A B} \cong \Gamma_{\ell-A, \ell-B}$ shows that for $A+B \leqslant \ell$ the space $\Gamma_{A B} / S_{+} \Gamma_{A-1, B-1}$ is the $g l(\ell)$ orbit of the vector

$$
v_{A B}=x_{1} x_{2} \ldots x_{A} \frac{\partial}{\partial x_{\ell-B+1}} \ldots \frac{\partial}{\partial x_{\ell}}
$$

with weight $n_{1}+\ldots+n_{A}-\left(n_{\ell-B+1}+\ldots+n_{\ell}\right)$. We have thus the decomposition of the Clifford algebra $C(\ell)$ into highest-weight modules $\mathcal{H}_{A B}=g l(\ell) \cdot v_{A B}$ of dimension $\operatorname{dim} \mathcal{H}_{A B}=\left(\begin{array}{l}\ell \\ A\end{array}\right)\left(\begin{array}{l}\ell \\ B\end{array}\right)-\left(\begin{array}{c}\ell \\ A-1\end{array}\right)\left(\begin{array}{c}\ell \\ B-1\end{array}\right)$,

$$
C(\ell)=\bigoplus_{A+B \leqslant \ell}\left(1+S_{+}\right)^{\ell-(A+B)}\left(\mathcal{H}_{A B} \oplus \mathcal{H}_{B A}\right)
$$


If we denote by $\nu_{A B}=\ell+1-(A+B)$ the multiplicity of the weight with indices $A B$ and by $f(t)$ the analytic part of the function $\left(t-t^{-1}\right)(1+t)^{\ell}\left(1+t^{-1}\right)^{\ell}$, i.e.

$$
\left(t-t^{-1}\right)(1+t)^{\ell}\left(1+t^{-1}\right)^{\ell}=f(t)-f\left(t^{-1}\right),
$$

the character formula

$$
\sum_{A+B \leqslant \ell} t^{\ell-(A+B)} \nu_{A B} \operatorname{dim} \mathcal{H}_{A B}=\frac{d}{d t} f(t)
$$

specialized at $t=1$ confirms that in fact $\sum \nu_{A B} \operatorname{dim} \mathcal{H}_{A B}=4^{\ell}=\operatorname{dim} C(\ell)$.

Let us denote by $\xi_{i j}=x_{i} \partial / \partial x_{j}$ the action of the elementary matrix $E_{i j}$ on $\Lambda(E)$. The Casimir operator of $g l(\ell)$,

$$
\Delta(X)=-\frac{1}{2} \sum_{i, j=1}^{\ell}\left[\xi_{i j},\left[\xi_{j i}, X\right]\right],
$$

can be written in terms of the (square of the) total spin operator

$$
S^{2}=S_{z}^{2}+\frac{1}{2}\left(S_{+} S_{-}+S_{-} S_{+}\right)
$$

and the number of particles operator $N$ as (see Ref. [8])

$$
\Delta=S^{2}+\frac{1}{4}(N-\ell)^{2}-\frac{\ell}{2}\left(\frac{\ell}{2}+1\right) \text {. }
$$

For $A+B \leqslant \ell$ this operator has an eigenvalue in the orbit of $v_{A B}$ given by

$$
\Delta\left(v_{A B}\right)=-\frac{1}{2}[\ell(A+B)-A(A-1)-B(B-1)] v_{A B},
$$

which in turn determines an eigenvalue $s(s+1)$ of $S^{2}$ with

$$
s=\frac{1}{2}(\ell-A-B) \text {. }
$$

This equation gives us the physical meaning of $A$ and $B$ for the modules $\Gamma_{A B}$ with $A+B \leqslant \ell: A-B+\ell$ is the total number of particles and $(\ell-A-B) / 2$ is the lowest spin attained in the module $\mathcal{H}_{A B}$, and the multiplicity $\nu_{A B}=2 s+1$.

\subsection{Spectral symmetries}

Let $X$ be an eigenstate of $H(U)$ with energy $E$,

$$
[K, X]+\frac{U}{2} \sum_{i=1}^{\ell}\left[n_{i},\left[n_{i}, X\right]\right]-\frac{\ell U}{4} X=E X .
$$

The adjoint of this equation is

$$
\left[K, X^{+}\right]-\frac{U}{2} \sum_{i=1}^{\ell}\left[n_{i},\left[n_{i}, X^{+}\right]\right]+\frac{\ell U}{4} X^{+}=-E X^{+},
$$


because $K$ and $n_{i}$ are self-adjoint operators; therefore $X^{+}$is an eigenstate of $H(-U)$ with eigenvalue $-E$. That is to say, the antilinear involution $X \rightarrow X^{+}$between $\Gamma_{A B}$ and $\Gamma_{B A}$ induces an inversion $(U, E) \rightarrow(-U,-E)$ of the energy levels in the $(U, E)$ plane. It can be easily seen that this transformation represents physically the exchange of pairs and holes.

A second spectral symmetry of interest is characteristic of those hopping matrices $t$ that admit a diagonal involution $\phi$ of the space $E\left(\phi x_{i}=\phi_{i} x_{i}, \phi^{2}=1\right)$ for which $\phi t \phi=-t$, as is the case of bipartite graphs. These unitary involutions induce also an inversion of the energy levels in the $(U, E)$ plane, but the module $\Gamma_{A B}$ is now preserved.

Finally, an involution $\phi$ can be combined with the Hodge dual $*: \Lambda(E) \rightarrow \Lambda(E)$, $u \rightarrow * u=u^{+} \Omega$, where $\Omega$ is a volume element in $A^{\ell}(E)$ with $(\Omega, \Omega)=1$, to define a linear transformation $\sigma: \Gamma_{A B} \rightarrow \Gamma_{\ell-A, B}$ by

$$
\sigma\left(x^{\alpha} \frac{\partial^{\beta}}{\partial x^{\beta}}\right)=\left(* x^{\alpha}\right) \phi\left(\frac{\partial^{\beta}}{\partial x^{\beta}}\right) .
$$

It can be checked that $\sigma$ anticommutes both with the kinetic-energy and potential-energy operators, and consequently

$$
\sigma H+H \sigma=0
$$

Therefore $\sigma$ induces a reflection $(U, E) \rightarrow(U,-E)$ in the $(U, E)$ plane. If $\ell$ is even and $X \in \Gamma_{\ell / 2, \ell / 2}$ is the image by $S_{+}^{\ell / 2-A}$ of an eigenstate in $\Gamma_{A A}$ with $A \leqslant \ell / 2, X$ contains at most $A$ pairs and $A$ holes. On the other hand, the eigenstate $\sigma(X) \in \Gamma_{\ell / 2, \ell / 2}$ with energy level $(U,-E)$ is made up from vectors with at least $\ell / 2-A$ pairs and $\ell / 2-A$ holes.

The relation between these transformations and the isospin algebra defined by Yang $[5,9]$ is the following: the action of Yang's $\eta$ operator in our representation is $\eta$ : $\Gamma_{A B} \rightarrow \Gamma_{A-1, B+1}$,

$$
\eta(X)=(-1)^{\ell+A-B} \sum_{i=1}^{\ell} \phi_{i} \frac{\partial}{\partial x_{i}} X \frac{\partial}{\partial x_{i}},
$$

and for states $X \in \Gamma_{A B}$ the automorphism $\sigma$ transforms $S_{+}$into

$$
\sigma S_{+} \sigma^{-1}=(-1)^{A+1} \eta
$$

\section{Non-trivial half-filled states in periodic lattices}

\subsection{Periodic lattices}

We restrict now our attention to $d$-dimensional periodic lattices with $\ell=\ell_{1} \times \ell_{2} \times$ $\ldots \times \ell_{d}$ sites. Each site $a=\left(a_{1}, \ldots, a_{d}\right)=a_{1} e_{1}+\ldots+a_{d} e_{d} \in L$ is a $d$-dimensional vector with coordinates $a_{j} \in \mathbb{Z}_{\ell_{j}}$ (the integers modulo $\ell_{j}$ ), and the space of states of a spinless particle is $E=\mathbf{C}\left\{x_{a}\right\}_{a \in L}$. The hopping matrix is 


$$
t=\sum_{j=1}^{d}\left(\tau_{j}+\tau_{j}^{-1}\right)
$$

where $\tau_{j} x_{a}=x_{a+e_{j}}, \tau_{j}^{\ell_{j}}=1$, and its extension to the exterior algebra $\Lambda(E)$ is given by

$$
K=\sum_{a \in L} t\left(x_{a}\right) \frac{\partial}{\partial x_{a}} .
$$

We transform to the momentum basis $\mathcal{F}=\left\{u_{m}\right\}_{m \in L}$, where

$$
u_{m}=\frac{1}{\sqrt{\ell}} \sum_{a \in L} x_{a}\left(\prod_{j=1}^{d} \epsilon_{j}^{-m_{j} a_{j}}\right)
$$

and $\epsilon_{j}=\exp \left(2 \pi i / \ell_{j}\right)$. In this representation the operator $K$ is diagonal,

$$
K=\sum_{m \in L} k_{m} u_{m} \frac{\partial}{\partial u_{m}},
$$

with eigenvalues

$$
k_{m}=\sum_{j=1}^{d}\left(\epsilon_{j}^{m_{j}}+\epsilon_{j}^{-m_{j}}\right)=2 \sum_{j=1}^{d} \cos \left(\frac{2 \pi m_{j}}{\ell_{j}}\right),
$$

the generators $n_{a}$ of $g l_{0}$ can be written as

$$
n_{a}=\frac{1}{\ell} \sum_{h \in L}\left(\prod_{j=1}^{d} \epsilon_{j}^{h_{j} a_{j}}\right) \theta_{h},
$$

where

$$
\theta_{h}=\sum_{m \in L} u_{m+h} \frac{\partial}{\partial u_{m}},
$$

and the expression of the Casimir operator $-\Delta_{0}$ in terms of the generators $\theta_{h}$ is

$$
\Delta_{0}(X)=\frac{1}{2 \ell} \sum_{h \in L}\left[\theta_{h},\left[\theta_{-h}, X\right]\right] .
$$

The Fourier transform exchanges the actions of the kinetic- and potential-energy operators: in the $x$-representation $K$ is determined by the abelian subalgebra $\tau^{a}$ and $-\Delta_{0}$ is associated to the diagonal subalgebra $g l_{0}$. In the $u$-representation $K$ is diagonal and $-\Delta_{0}$ is the Casimir operator of $\tau^{h}$. The Schrödinger equation for the hamiltonian (2.1) in the momentum representation is

$$
\sum_{h \in L}\left(k_{h}\left[u_{h} \frac{\partial}{\partial u_{h}}, X\right]+\frac{U}{2 \ell}\left[\theta_{h},\left[\theta_{-h}, X\right]\right]-\frac{\ell U}{4} X\right)=E X .
$$

We will not make use of the full spatial symmetry of the periodic lattice [11], only of the translational symmetry: the hamiltonian commutes with the unitary operators $\tau_{j}$ and 
the action $\tau_{j} u_{h}=\epsilon_{j}^{h_{j}} u_{h}$ shows that the modules $\Gamma_{A B}$ can be further decomposed into invariant modules

$$
\Gamma_{A B}=\bigoplus_{h \in L} \Gamma_{A B}^{h},
$$

where

$$
\Gamma_{A B}^{h}=\mathbf{C}\left\{X \in \Gamma_{A B}: \tau_{j} X=\epsilon_{j}^{h_{j}} X\right\} .
$$

\subsection{Half-filled states with spin $(\ell-2) / 2$}

In this subsection we solve explicitly the Schrödinger equation (3.1) in the simplest module (except for the $\Gamma_{A 0}$ and $\Gamma_{0 B}$ ), $\Gamma_{11}=g l(\ell)=s l(\ell) \oplus \mathbf{C n}$. We seek the solutions $X \in \Gamma_{11}^{h}$ by writing

$$
X=\sum_{m \in L} c_{m} \xi_{m}
$$

where we have defined

$$
\xi_{m}=u_{m+h} \frac{\partial}{\partial u_{m}}
$$

and to simplify the notation we omit the label $h$ in most of the magnitudes. The substitution of Eq. (3.2) into the Schrödinger equations (3.1) gives

$$
\sum_{m \in L}\left(\omega_{m}-s\right) c_{m} \xi_{m}=\frac{U}{\ell} \sum_{m \in L}\left(\sum_{n \in L} c_{n}\right) \xi_{m},
$$

where $\omega_{m}=k_{m+h}-k_{m}$ are the eigenvalues of $T$ and $s=E-(1-\ell / 4) U$. Eq. (3.3) is equivalent to the following system of linear equations for the unknown coefficients $c_{m}$ :

$$
\left(\omega_{m}-s\right) c_{m}=\frac{U}{\ell} \sum_{n \in L} c_{n},
$$

and shows that if $g_{m}$ is the degeneracy of the unperturbed eigenvalue $\omega_{m}$, then from $\omega_{m}$ stem exactly $g_{m}-1$ degenerate eigenvalues $s=\omega_{m}$ or

$$
E=\omega_{m}+(1-\ell / 4) U
$$

and one eigenvalue explicitly given by

$$
\begin{aligned}
& \frac{\ell}{U(s)}=\sum_{m \in L} \frac{1}{\omega_{m}-s}, \\
& E(s)=s+(1-\ell / 4) U(s) .
\end{aligned}
$$

The $g_{m}-1$ eigenvectors corresponding to $s=\omega_{m}$ have coefficients $c_{k}$ such that $c_{k}=0$ if $\omega_{m} \neq \omega_{k}, \sum_{k} c_{k}=0$ where the sum runs over those $k$ with $\omega_{m}=\omega_{k}$, and one of 
the coefficients is fixed by normalization. The remaining eigenvectors (one for each different unperturbed eigenvalue) are

$$
X(s)=\frac{1}{\left\|(T-s)^{-1} \theta_{h}\right\|}(T-s)^{-1} \theta_{h},
$$

and the normalization constant has a convenient expression in terms of the characteristic polynomial of $T$ restricted to $\Gamma_{11}^{h}$,

$$
p(s)=\operatorname{det}(T-s)=\prod_{m \in L}\left(\omega_{m}-s\right) .
$$

In fact, Eq. (3.6) can be rewritten as $\ell / U(s)=-d / d s \ln p(s)$ and, with the exception of $h=0$ explained below,

$$
\left\|(T-s)^{-1} \theta_{h}\right\|^{2}=-2^{\ell-2} \frac{d^{2}}{d s^{2}} \ln p(s) .
$$

For later reference we quote the explicit formula for these normalized eigenvectors $X(s)$ :

$$
X(s)=\frac{2^{1-\ell / 2} p(s)}{\left(\dot{p}(s)^{2}-p(s) \ddot{p}(s)\right)^{1 / 2}} \sum_{m \in L} \frac{1}{\omega_{m}-s} \xi_{m} .
$$

We mention that these "generic" eigenvalues can be considered as a unique curve that wraps around a Riemann sphere as many times as there are different unperturbed eigenvalues. More precisely, the generic state (3.10) is a section of a line bundle on the Riemann sphere over the curve $(U(s), E(s))$, and the different energy levels are the projections of that curve around the origin.

Incidentally, the characteristic polynomial of the system of Eqs. (3.4) can be explicitly calculated,

$$
P(s)=p(s)+\frac{U}{\ell} \dot{p}(s),
$$

and provides an alternative path to obtain the solutions (3.5) and (3.7).

Let us study in detail now the special case $h=0$, in which all the kinetic-energy eigenvalues $\omega_{m}$ vanish. We stress that the following considerations are independent of the dimension or "shape" of the lattice, but depend only on the periodicity. Eq. (3.6) simplifies to $U=-s$ and the corresponding normalized eigenvector (3.8) is a multiple of the identity

$$
X=\frac{1}{\sqrt{\ell(\ell+1) 2^{\ell-2}}} n .
$$

The eigenvectors associated to the remaining $\ell-1$ degenerate eigenvalues can be taken to be any of the $\ell-1$ linearly independent solutions of $\sum_{m \in L} c_{m}=0$, with one of the coefficients arbitrarily fixed to 1 . The standard (unnormalized) choice is

$$
X_{m}=\xi_{m}-\xi_{m+1} \quad(m=0, \ldots, \ell-2),
$$

and precisely in the subspace spanned by these vectors we find the special eigenstate $K$ that we studied in Ref. [8]. It is worth noting also that these two families of 
solutions (3.11) and (3.12) correspond to the decomposition of the matrices in the $\Gamma_{11}^{0}$ module into a multiple of the identity and a traceless matrix.

The norm of a generic state $X=\sum_{i, j=1}^{\ell} c_{i j} u_{i} \partial / \partial u_{j}$ in $\Gamma_{11}$ is given by

$$
\|X\|^{2}=\operatorname{tr}\left(X^{+} X\right)=2^{\ell-2}\left(\|c\|^{2}+|\operatorname{tr} c|^{2}\right),
$$

where $\|c\|^{2}=\sum_{i, j=1}^{\ell}\left|c_{i j}\right|^{2}$, and the expectation value of $S_{z}$ is

$$
\left\langle S_{z}\right\rangle_{X}=\frac{|\operatorname{tr} c|^{2}}{\|c\|^{2}+|\operatorname{tr} c|^{2}}
$$

From our previous discussion it follows that $n$ is the only eigenstate of the Hubbard hamiltonian in $\Gamma_{11}$ for which $\operatorname{tr} c \neq 0$; hence the exception in the normalization quoted above, and the fact that it is the only eigenstate in $\Gamma_{11}$ with a non-vanishing expectation value of $S_{z}\left[\left\langle S_{z}\right\rangle_{n}=\ell /(\ell+1)\right]$. Eq. (2.2) and a simple homogeneity argument also show that any state in a $\Gamma_{A B}$ module has a vanishing expectation value of Yang's $\eta$ operator.

As a second example we study the $h=(1,0)$ sector of a two-dimensional square lattice with sixteen sites, i.e. we take $d=2$ and $\ell=4 \times 4$, so that $s=E+3 U$. The unperturbed eigenvalues $\omega_{m}$ are -2 and 2 , both with multiplicity 8 , and therefore $p(s)=\left(s^{2}-4\right)^{8}$. Eq. (3.6) is now

$$
U(s)=-s+\frac{4}{s}
$$

which has two solutions for each value of $U$, and the corresponding normalized eigenvectors (3.10) are

$$
X(s)=-\frac{1}{2^{9}}\left[\frac{s-2}{\sqrt{s^{2}+4}} \sum_{\omega_{m}=-2} \xi_{m}+\frac{s+2}{\sqrt{s^{2}+4}} \sum_{\omega_{m}=2} \xi_{m}\right] .
$$

Note that at $s=2$ and $s=-2, X(2)$ and $X(-2)$ are eigenvectors of $T=H$, because $U(2)=U(-2)=0$, and at $s= \pm \infty$,

$$
X( \pm \infty)=\mp \frac{1}{2^{9}} \theta_{(1,0)}
$$

are eigenvectors of the potential energy $W$. Besides these two states and because of the eightfold degeneracy of the unperturbed eigenvalues, we have also the seven linear states with $s=\omega_{m}=-2$ and the seven with $s=\omega_{m}=2$, whose eigenvectors are proportional to $\xi_{m}-\xi_{m+1}$ in the respective degenerate subspaces.

\subsection{A second description of the energy levels in the sl( $\ell)$ module}

In Subsection 3.2 we obtained states in $\Gamma_{11}$ acting with the resolvent $(T-s)^{-1}$ on vectors $\theta_{h}$ in a Cartan subalgebra of $s l(\ell)$ with potential energy $-\ell / 4$. With the introduction of a new parameter $\tilde{s}=E+\ell U / 4$, these same states can be equivalently 
characterized by the action of $(T-\tilde{s})^{-1}$ on the image under the adjoint action of the Cartan subalgebra, with potential energy $(1-\ell / 4)$.

In terms of the new parameter $\tilde{s}$, Eq. (3.3) can be rewritten

$$
\sum_{m \in L}\left(\omega_{m}-\tilde{s}\right) c_{m} \xi_{m}=U \sum_{m \in L} q_{m} \xi_{m}
$$

where

$$
q_{m}=-c_{m}+\frac{1}{\ell} \sum_{n \in L} c_{n} .
$$

For the above-mentioned states with $\sum_{n \in L} c_{n} \neq 0$, the compatibility condition of the previous equation is the "adjoint" condition $\sum_{m \in L} q_{m}=0$, and the new coefficients are

$$
q_{m}=\frac{\omega_{m}-\tilde{s}}{\omega_{m}-\tilde{s}+U(\tilde{s})}
$$

with the function $U(\tilde{s})$ determined by

$$
\frac{\ell}{U(\tilde{s})}=\sum_{m \in L} \frac{1}{\omega_{m}-\tilde{s}+U(\tilde{s})} .
$$

The full solution is, as we anticipated,

$$
X(\tilde{s})=(T-\tilde{s})^{-1} \sum_{m \in L} q_{m} \xi_{m},
$$

and the equivalence between Eqs. (3.13) and (3.6) is obvious, because $\tilde{s}=s+U$.

For example, consider the case $d=1, \ell=4$, and $h=2$. The unperturbed eigenvalues are $\omega_{0}=-\omega_{2}=-4$ and $\omega_{1}=\omega_{3}=0$. In our first parametrization $E=s$, we have

$$
U(s)=s\left(s^{2}-16\right) /\left(8-s^{2}\right)
$$

which provides a rational parametrization of the cubic

$$
E^{3}+U E^{2}-16 E-8 U=0 .
$$

In our second parametrization, $E=\tilde{s}-U$, we have

$$
\tilde{s} U^{2}-2\left(\tilde{s}^{2}-4\right) U-16 \tilde{s}+\tilde{s}^{3}=0,
$$

which describes the curve with

$$
U_{ \pm}=\frac{\tilde{s}^{2}-4 \mp 2 \sqrt{2\left(2+\tilde{s}^{2}\right)}}{\tilde{s}} .
$$

The appearance of two branches $\left(U_{+}, E_{+}\right),\left(U_{-}, E_{-}\right)$reflects the fact that the subspace $\operatorname{im} \Delta_{0} \cap \Gamma_{11}^{2}$, which complements $\mathbf{C} \theta_{2}$ in $\Gamma_{11}^{2}=\mathbf{C} \theta_{2} \oplus\left(\mathrm{im} \Delta_{0} \cap \Gamma_{11}^{2}\right)$, contains two independent vectors that are not common eigenvectors of $T$ and $\Delta_{0}$ (the common eigenvector is $\left.\xi_{1}-\xi_{3}\right)$. Fig. 1 illustrates these two parametrizations of the same curve. In Fig. 1a the three energy levels correspond to the intervals $(-\infty,-\sqrt{8}),(-\sqrt{8}, \sqrt{8})$ 

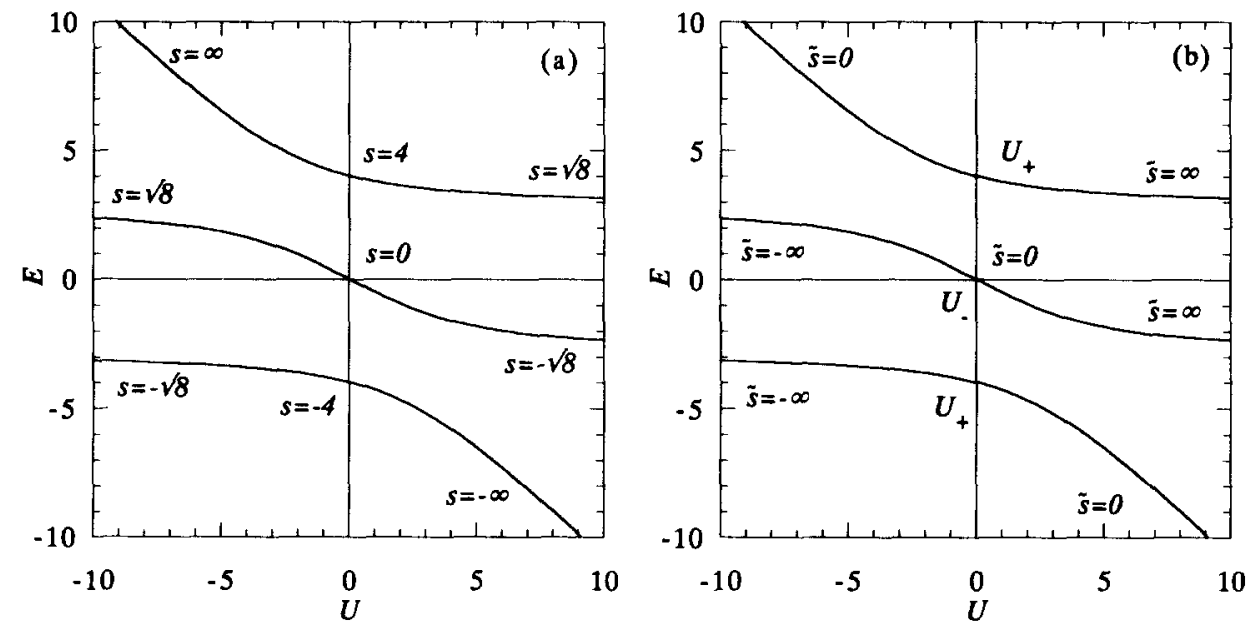

Fig. 1. Two parametrizations of the non-linear eigenvalues in the $h=2$ sector of a one-dimensional half-filled periodic lattice with four sites.

and $(\sqrt{8}, \infty)$ of a single curve that wraps three times around the Riemann sphere as the parameter $s$ goes from $-\infty$ to $\infty$. Note also that in each of the intervals lies one of the values $s=\omega_{m}(-4,0$, and 4 respectively $)$ at which $U=0$. The second parametrization is shown in Fig. $1 \mathrm{~b}$, and the three energy levels appear now as the two curves $U_{+}$and $U_{-}$defined above, one of which $\left(U_{+}\right)$wraps twice around the Riemann sphere as the parameter $\tilde{s}$ goes from $-\infty$ to $\infty$.

\section{General description of the space of states}

\subsection{The structure of the space of states}

In this section we denote by $\mathcal{E}$ any Hilbert subspace of $\mathcal{H}$ stable under the action of both the kinetic- and potential-energy operators (for example, the modules $\mathcal{H}_{A B}$ in $C(\ell))$. We associate to each eigenvalue $\lambda$ of the self-adjoint operator $\Delta_{0}$ in $\mathcal{E}$ the direct-sum decomposition into orthogonal subspaces

$$
\mathcal{E}=\mathcal{E}_{\lambda} \oplus \mathcal{E}_{\lambda}^{\perp}
$$

(where $\Delta_{0}=\lambda$ in $\mathcal{E}_{\lambda}$ ) and define a parameter $s$ by

$$
E=s+(\lambda-\ell / 4) U \text {. }
$$

For any fixed $\lambda \in \sigma_{\mathcal{E}}\left(\Delta_{0}\right)$, the eigenvalues and eigenvectors of $H$ in $\mathcal{E}$ are the solutions of

$$
(T-s) X=U\left(\lambda-\Delta_{0}\right) X
$$


where we have added the vector $\lambda U X$ to both sides of the original Schrödinger equation. That is, for each value of $s, U$ is an eigenvalue of $T-s$ relative to $\lambda-\Delta_{0}$. Note that the vector $Y=\left(\lambda-\Delta_{0}\right) X$ is contained in the subspace $\mathcal{E}_{\lambda}^{\perp}$. Therefore, in the generic situation $s \notin \sigma_{\mathcal{E}}(T)$ we can obtain an equation for $Y \in \mathcal{E}_{\lambda}^{\perp}$,

$$
U^{-1} Y=\left(\lambda-\Delta_{0}\right)(T-s)^{-1} Y
$$

We stress that Eqs. (4.1) and (4.2) for $X$ and $Y$ are equivalent whenever the following two conditions are met: (i) $s \notin \sigma_{\mathcal{E}}(T)$, i.e. out of the subspaces corresponding to linear eigenvalues $s \in \sigma_{\mathcal{E}_{\lambda}}(T)$, in which $E=s+(\lambda-\ell / 4) U$ and $(T-s) X=\left(\lambda-\Delta_{0}\right) X=$ 0 ; and (ii) $U^{-1}$ is a real eigenvalue of the (in general not self-adjoint operator) $\left(\lambda-\Delta_{0}\right)(T-s)^{-1}$ in $\mathcal{E}_{\lambda}^{\perp}$. We will see next that this is the case for the extremal eigenvalues $\lambda_{+}$and $\lambda_{-}$of $\Delta_{0}$ in $\mathcal{E}\left(\lambda_{-} \leqslant \sigma_{\mathcal{E}}\left(\Delta_{0}\right) \leqslant \lambda_{+}\right)$.

Let us consider the case of $\lambda=\lambda_{+}$in Eq. (4.2). Since $\lambda_{+}-\Delta_{0}$ is positive definite in $\mathcal{E}_{\lambda}^{\perp}$, we can define

$$
\begin{aligned}
D_{+} & =\left(\lambda_{+}-\Delta_{0}\right)^{1 / 2}, \\
Z & =\frac{1}{\left\|D_{+}^{-1} Y\right\|} D_{+}^{-1} Y,
\end{aligned}
$$

and rewrite Eq. (4.2) as follows:

$$
U^{-1} Z=D_{+}(T-s)^{-1} D_{+} Z .
$$

Therefore $U^{-1}$ is indeed a (real) eigenvalue of a self-adjoint operator in $\mathcal{E}_{\lambda}^{\perp}$. A similar factorization of $\Delta_{0}-\lambda_{-}$reduces Eq. (4.2) to

$$
U^{-1} Z=-D_{-}(T-s)^{-1} D_{-} Z,
$$

where $D_{-}=\left(\Delta_{0}-\lambda_{-}\right)^{1 / 2}$, and $Z=\left\|D_{-}^{-1} Y\right\|^{-1} D_{-}^{-1} Y$.

\subsection{Energy bands}

If we multiply Eq. (4.3) by $s$ and take the limit $s \rightarrow \infty$, we find that the normalized vector $Z(\infty)$ is an eigenvector of the shifted potential $\lambda_{+}-\Delta_{0}=D_{+}^{2}$ corresponding to an eigenvalue

$$
\lim _{s \rightarrow \infty}\left(s U^{-1}\right)=-\lambda_{+}+\mu, \quad \mu \in \sigma_{\mathcal{E}_{\lambda_{+}}^{\perp}}\left(\Delta_{0}\right),
$$

or, equivalently, to

$$
\lim _{s \rightarrow \infty}\left(E U^{-1}\right)=\mu-\ell / 4 .
$$

Our next aim is to follow the evolution of $Z(\infty)$ and $U^{-1}$ as the parameter $s$ decreases from infinity. We take the scalar product of Eq. (4.3) with the normalized vector $Z(s)$,

$$
U_{Z}^{-1}=\left(Z, D_{+}(T-s)^{-1} D_{+} Z\right)=\left(D_{+} Z,(T-s)^{-1} D_{+} Z\right)
$$


Table 1

Characteristic polynomials of a half-filled periodic lattice with four sites

\begin{tabular}{ll}
\hline$\Gamma_{A B}^{h}$ & $P(U, E)$ \\
\hline$\Gamma_{11}^{3}$ & $E^{3}(E+U)$ \\
$\Gamma_{11}^{1} \cong \Gamma_{11}^{3}$ & $(-2+E)(2+E)\left(-4+E^{2}+E U\right)$ \\
$\Gamma_{11}^{2}$ & $E\left(-16 E+E^{3}-8 U+E^{2} U\right)$ \\
$\Gamma_{22}^{0}$ & $E^{3}(E+U)\left(16 E-E^{3}-8 U+E^{2} U\right)\left(16 E-E^{3}-8 U+E U^{2}\right)$ \\
$\Gamma_{22}^{1} \cong \Gamma_{22}^{3}$ & $(-2+E)^{2}(2+E)^{2}\left(4-E^{2}-E U\right)\left(4-E^{2}+E U\right)$ \\
$\Gamma_{22}^{2}$ & $E^{3}(E-U)\left(16 E-E^{3}+8 U-E^{2} U\right)\left(16 E-E^{3}+8 U+E U^{2}\right)$ \\
\hline
\end{tabular}

and expand

$$
\frac{1}{\left\|D_{+} Z\right\|} D_{+} Z=\sum_{m} \zeta_{m}(s) v_{m},
$$

where $\left\{v_{m}\right\}$ is a basis of normalized eigenvectors of $T$ in $\mathcal{E}$, with associated eigenvalues $\omega_{m}$. Note that $\sum_{m}\left|\zeta_{m}(s)\right|^{2}=1$, and $\left\|D_{+} Z\right\|^{2}=\left\langle\lambda_{+}-\Delta_{0}\right\rangle_{Z}$ is a bounded quantity. Therefore,

$$
\frac{1}{U_{Z}(s)}=\left\langle\lambda_{+}-\Delta_{0}\right\rangle_{Z(s)} \sum_{m} \frac{\left|\zeta_{m}(s)\right|^{2}}{\omega_{m}-s},
$$

with $0 \leqslant\left|\zeta_{m}(s)\right| \leqslant 1$. As a matter of fact, Eq. (3.6) is a particular case of Eq. (4.4) with $\lambda_{+}=1, Z(s)=\left(2^{\ell-2} \ell\right)^{-1 / 2} \theta_{h}, \zeta_{m}(s)=\ell^{-1 / 2}$, and $\left\langle\lambda_{+}-\Delta_{0}\right\rangle_{Z(s)}=1$, and the generic behavior of $U_{Z}(s)$ is analogous to the behavior we discussed in the examples. Between any two consecutive frequencies $\omega_{m}$ and $\omega_{m+1}$ there is a value of $s$ for which $U \rightarrow \infty$ or $-\infty$ (alternatively), while the $s=\omega_{m}$ correspond to $U=0$ and $E \rightarrow \infty$ with slope $\lambda_{+}-\ell / 4$ (remember that $E=s+\left(\lambda_{+}-\ell / 4\right) U$ ). The possibility of connecting the slope $\mu$ with $\lambda_{+}-\ell / 4$ is determined by the appearance of more than one frequency in the expression of $D_{+} Z$. That this number is generically greater than one follows from the fact that $\Delta_{0}$ and $T$ are not simultaneously diagonalizable.

As we have seen, each level $\mu$ spreads in a band under the action of $T-s$. What distinguishes the aspect of these bands for the Hubbard hamiltonian from other possible choices of $T$ is the definition of $T$ as the representation of a hopping matrix. The number of connected levels corresponds to the spaces $\mathcal{E}_{\lambda}$ that can be taken as the irreducible modules. Each eigenvalue $\lambda$ of $\Delta_{0}$ defines a space $\mathcal{E}_{\lambda}$. At the level $\mu$ starts a curve associated to each of the eigenvectors $Z(\infty)$ of the potential energy that propagates according to Eq. (4.3) and produces the state $X$. These remarks are illustrated in Fig. 2, which shows all the energy levels at half filling of a periodic one-dimensional lattice with four sites. Table 1 also gives the independent characteristic polynomials that describe analytically the curves plotted in Fig. 2 (the characteristic polynomials of higher modules are not displayed because $\Gamma_{A A} \cong \Gamma_{\ell-A, \ell-A}$.). Incidentally, we can see 


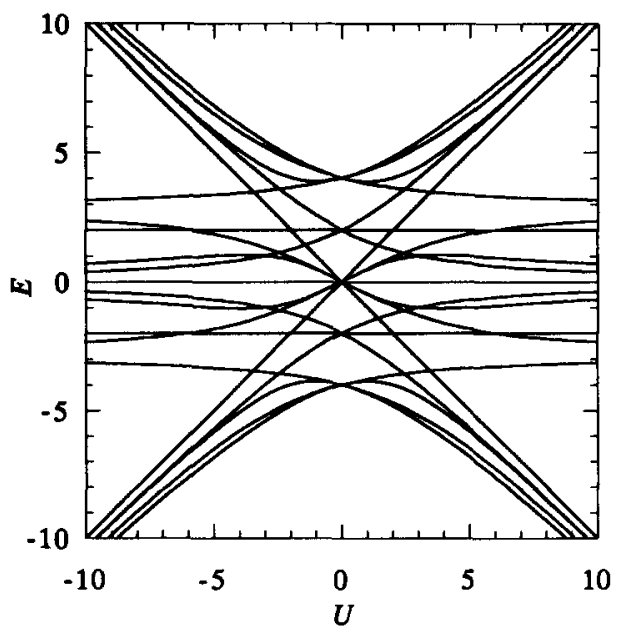

Fig. 2. Half-filled energy levels of a one-dimensional periodic lattice with four sites.

also how each module $\Gamma_{A A}^{h}$ contains an image of the module $\Gamma_{A-1, A-1}^{h}$, and the effect of both spectral symmetries discussed in Subsection 2.3.

\section{References}

[1] D.C. Mattis, ed., The many-body problem (World Scientific, Singapore, 1993).

[2] V.E. Korepin and F.H. Eßler, eds., Exactly solvable models of strongly correlated electrons (World Scientific, Singapore, 1994).

[3] E.H. Lieb, in Proceedings of the XIth International Congress of Mathematical Physics, ed. D. Iagolnitzer (International Press, Boston, 1995).

[4] E.H. Lieb and F.Y. Wu, Phys. Rev. Lett. 20 (1968) 1445.

[5] C.N. Yang, Phys. Rev. Lett. 63 (1989) 2144.

[6] E.H. Lieb, Phys. Rev. Lett. 62 (1989) 1021.

[7] A. Mielke and H. Tasaki, Commun. Math. Phys. 158 (1993) 341.

[8] F. Guil and G. Alvarez, Nuc. Phys. B 458 (1996) 609.

[9] C.N. Yang and S.C. Zhang, Mod. Phys. Lett. B 4 (1990) 759.

[10] W. Fulton and J. Harris, Representation Theory (Springer, Berlin, 1991).

[11] G. Fano, F. Ortolani and A. Parola, Phys. Rev. B 46 (1992) 1048. 